\title{
METRIZABILITY OF DECOMPOSITION SPACES
}

\author{
A. H. STONE
}

1. Introduction. Let $f$ be a quasicompact continuous mapping of a metric space $S$ onto a topological space $E$; that is, let $f$ be a mapping of $S$ onto $E$ such that a subset $Y$ of $E$ is open (closed) in $E$ if and only if $f^{-1}(Y)$ is open (closed) in $S$. As is well known, ${ }^{1} E$ is then homeomorphic, in a natural way, to the hyperspace of the decomposition of $S$ into the disjoint nonempty sets $F_{p}=f^{-1}(p)(p \in E)$, while conversely the hyperspace of every decomposition of $S$ into disjoint nonempty sets $F_{p}$ arises in this way, essentially uniquely. The object of this paper is to determine conditions under which $E$ will be metrizable. Results of this nature have been known for some time, particularly for the special case in which $f$ is closed (i.e., $f(X)$ is closed in $E$ whenever $X$ is closed in $S$ ) -or, equivalently, the case in which the decomposition $\left\{F_{p}\right\}$ is upper semi-continuous (i.e., if $F_{p} \subset U$ where $U$ is open, there exists an open set $V$ such that $F_{p} \subset V$ and every $F_{q}$ meeting $V$ is contained in $\left.U\right) .{ }^{2}$ But it seems to have escaped notice that the problem has a simple complete answer in this case (Theorem 1), without any assumptions of compactness or separability. ${ }^{3}$ We shall also improve some known results about quasicompact images of locally compact spaces (Theorems 2 and 3), and obtain a criterion (Theorem 4 ) for the metrizability of $E$ when $f$ is open (i.e., when the decomposition is lower semi-continuous). Here the conditions we obtain are sufficient but not necessary; however, we show by examples that they are not superfluous. Finally we apply Theorem 1 to obtain a simple description of the case in which $f$ is both open and closed (i.e., when the decomposition is continuous); it turns out that $E$ is then always metrizable, with a quite convenient metric.

\section{Closed mappings.}

Received by the editors April 22, 1955.

${ }^{1}$ See $[2$, p. $65 ; 5 ; 10]$. In the terminology of $[2]$ "quasicompact continuous" = "stark-stetig." In the terminology of Bourbaki, $E$ is the space $S / R$ where $R$ is the equivalence relation corresponding to $f$ (i.e., $x R y \leftrightarrow f(x)=f(y)$ ).

2 See $[9$, p. $123 ; 4]$. The term "upper semi-continuous decomposition" has been used in the literature with several different meanings, but that given above seems to be generally adopted now. It is equivalent to "stetig Zerlegung" in [2], except that we do not require $F_{p}$ to be closed.

${ }^{3}$ Another, independent, proof of Theorem 1 has appeared after this paper was written; see K. Morita and S. Hanai, Closed mappings and metric spaces, Proc. Japan Acad. vol. 32 (1956) pp. 10-14. 
THEOREM 1. Let $f$ be a closed continuous mapping of a metric space $S$ onto a topological space $E$. Then the following statements are all equivalent: ${ }^{4}$

(i) E satisfies the first countability axiom.

(ii) For each $p \in E, f^{-1}(p)$ has a compact frontier (in $S$ ).

(iii) $E$ is metrizable.

REMARK. $E$ is in any case a $T_{1}$ space, for each $p \in E$ is of the form $f(x)$ where $(x)$ is closed. Hence $F_{p}=f^{-1}(p)$ is closed in $S$, for each $p \in E$.

It is enough to prove (i) $\rightarrow$ (ii) and (ii) $\rightarrow$ (iii).

(i) $\rightarrow$ (ii). Let $\left\{W_{n}(p)\right\}(n=1,2, \cdots)$ be a countable basis of open neighborhoods of $p \in E$. If $\mathrm{Fr}\left(F_{p}\right)$ is not compact, there is a sequence $\left\{x_{n}\right\}(n=1,2, \cdots)$ of points of $\operatorname{Fr}\left(F_{p}\right)$ having no cluster point in $\operatorname{Fr}\left(F_{p}\right)$, and consequently no cluster point in $S$. Now $f^{-1}\left(W_{n}(p)\right)$ is an open set containing $F_{p}$; as $F_{p}$ is closed, $x_{n} \in f^{-1}\left(W_{n}(p)\right)$, and so there exists $y_{n} \in S-F_{p}$ such that $y_{n} \in f^{-1}\left(W_{n}(p)\right)$ and $\rho\left(x_{n}, y_{n}\right)<1 / n$, $\rho$ denoting the distance in $S$. Let $Y=\left\{y_{n}\right\} ; Y$ is closed, since the sequence $\left\{y_{n}\right\}$ has no cluster point in $S$ (else the sequence $\left\{x_{n}\right\}$ would). Hence $Q=f(Y)$ must be closed in $E$. By construction, $p \notin Q$; yet $p \in \bar{Q}$ since $W_{n}(p)$ meets $Q$ in $f\left(y_{n}\right)$, and this contradicts the closedness of $Q$.

(ii) $\rightarrow$ (iii) The case in which the sets $F_{p}$ are themselves compact has been established by S. Hanai, ${ }^{5}$ and the present proof uses the same idea as his. We construct for each $p \in E$ a sequence $\left\{W_{n}(p)\right\}$ $(n=1,2, \cdots)$ of open sets and prove $(1) W_{1}(p) \supset W_{2}(p) \supset \cdots$, (2) $\bigcap_{n} W_{n}(p)=(p),(3)$ the sets $W_{n}(p)$ form a basis at $p,(4)$ given a positive integer $n$ and a point $p$ of $E$, there exists $m$ such that whenever $W_{m}(q)$ meets $W_{m}(p)$ we have $W_{m}(q) \subset W_{n}(p)$. By a theorem of Mrs. Frink [3], $E$ will then be metrizable.

For any set $X \subset S$, write $S(X, \epsilon)$ to denote the open $\epsilon$-neighborhood $\{y \mid \rho(y, X)<\epsilon\}$ of $X$. (If $X=\varnothing, S(X, \epsilon)=\varnothing$.) Now write $N_{p}^{n}$ $=S\left(\operatorname{Fr}\left(F_{p}\right), \quad 1 / n\right), \quad U_{p}^{n}=N_{p}^{n} \cup \operatorname{Int}\left(F_{p}\right), \quad V_{p}^{n}=\bigcup\left\{F_{q} \mid F_{q} \subset U_{p}^{n}\right\}, \quad$ and $W_{n}(p)=f\left(V_{p}^{n}\right)=\left\{q \mid F_{q} \subset U_{p}^{n}\right\}$. Thus $W_{n}(p)=E-f\left(S-U_{p}^{n}\right) ;$ and, as $U_{p}^{n}$ is open, it follows from the closedness of $f$ that $W_{n}(p)$ and so $V_{p}^{n}$ are open. Clearly $U_{p}^{n} \supset V_{p}^{n} \supset F_{p}$, so $W_{n}(p) \ni p$. The assertion (1) is trivial, and (2) follows from (3) since $E$ is $T_{1}$. To prove (3), let $G$ be any open set (in $E$ ) containing $p$; then $\operatorname{Fr}\left(F_{p}\right) \subset F_{p} \subset f^{-1}(G)$, and so the closed sets $\operatorname{Fr}\left(F_{p}\right), S-f^{-1}(G)$, are disjoint. As $\operatorname{Fr}\left(F_{p}\right)$ is com-

4 The implication (iii) $\rightarrow$ (ii) is due to Valnstern [7]. If $S$ is separable, the implication (i) $\rightarrow$ (iii) is implicit in [10].

5 See [4]; another proof is implicit in [11, p. 367]. The general case can be deduced from the $F_{p}$-compact case; but, as the proof in [4] has a gap and that in [11] uses different basic definitions, it seems preferable to give a direct argument. 
pact, the distance $d=\rho\left\{\operatorname{Fr}\left(F_{p}\right), S-f^{-1}(G)\right\}$ is positive. Take $n>1 / d$; then $N_{p}^{n} \subset f^{-1}(G)$, and consequently $f^{-1}(G) \supset U_{p}^{n} \supset V_{p}^{n}$, so that $G \supset W_{n}(p)$, q.e.d.

To prove (4), suppose $p$ and $n$ given. If Int $\left(F_{p}\right) \neq \varnothing$, pick a point $x_{p} \in \operatorname{Int}\left(F_{p}\right)$ arbitrarily. Now take $m$ so large that

(a) $m>2 n$,

(b) if $\operatorname{Fr}\left(F_{p}\right) \neq \varnothing, \rho\left(\operatorname{Fr}\left(F_{p}\right), S-V_{p}^{2 n}\right)>2 / m$,

(c) if Int $\left(F_{p}\right) \neq \varnothing, S\left(x_{p}, 1 / m\right) \subset F_{p}$.

Suppose $W_{m}(q)$ meets $W_{m}(p)$ in $r$, say, where we assume that $q \neq p$. We first show that $F_{q} \subset V_{p}^{2 n}$. Take $w \in F_{r} \subset V_{q}^{m} \cap V_{p}^{m} \subset U_{q}^{m} \cap U_{p}^{m}$. If $w \in \operatorname{Int}\left(F_{q}\right)$, then $F_{q}$ meets $V_{p}^{m}$ and consequently $F_{q} \subset V_{p}^{m} \subset V_{p}^{2 n}$ as required. Since $w \in U_{q}^{m}=N_{q}^{m} \cup$ Int $\left(F_{q}\right)$, we may assume now that $w \in N_{q}^{m}$. Again, if $w \in \operatorname{Int}\left(F_{p}\right)$, then $x_{p}$ exists; also $F_{p}$ meets $V_{q}^{m}$ and so $F_{p} \subset V_{q}^{m} ;$ hence $x_{p} \in V_{q}^{m} \subset$ Int $\left(F_{q}\right) \cup N_{q}^{m}$, and as $x_{p} \notin F_{q}$ (for $p \neq q$ ) we have $x_{p} \in N_{q}^{m}$, which violates condition (c). Hence $w \in N_{q}^{m} \cap N_{p}^{m}$, and there exist points $x \in \operatorname{Fr}\left(F_{p}\right), y \in \operatorname{Fr}\left(F_{q}\right)$, such that $\rho(w, x)<1 / m$ and $\rho(w, y)<1 / m$. It follows that $\rho(x, y)<2 / m$, and condition (b) shows that $y \in V_{p}^{2 n}$. As $F_{q}$ meets $V_{p}^{2 n}$, we have $F_{q} \subset V_{p}^{2 n}$.

Next we deduce $U_{q}^{m} \subset U_{p}^{n}$. The preceding argument has shown that $F_{q} \subset U_{p}^{2 n}=N_{p}^{2 n} \cup$ Int $\left(F_{p}\right)$; hence $F_{q} \subset N_{p}^{2 n}$. Let $z$ be any point of $U_{q}^{m}$; then there exists $y^{\prime} \in F_{q}$ such that $\rho\left(z, y^{\prime}\right)<1 / m<1 / 2 n$, from (a). Since $y^{\prime} \in N_{p}^{2 n}$, there exists $x^{\prime} \in \operatorname{Fr}\left(F_{p}\right)$ such that $\rho\left(x^{\prime}, y^{\prime}\right)<1 / 2 n$. Thus $\rho\left(x^{\prime}, z\right)<1 / n$, so that $z \in N_{p}^{n} \subset U_{p}^{n}$. This proves $U_{q}^{m} \subset U_{p}^{n}$ if $p \neq q$; but if $p=q$ then $U_{q}^{m} \subset U_{p}^{n}$ trivially. Hence $V_{q}^{m} \subset V_{p}^{n}$ in any case, whence $W_{m}(q) \subset W_{n}(p)$, and the proof is complete.

COROLLARY 1. If the conditions of the theorem are satisfied, and if $S$ is separable, or locally separable, or compact, or locally compact, then $E$ also has the corresponding property.

Compactness and separability are preserved by arbitrary continuous mappings. Suppose $S$ locally compact; we prove $E$ locally compact at $p \in E$. Since $\operatorname{Fr}\left(F_{p}\right)$ is compact, it is covered by a finite number of open sets with compact closures. Thus, if $n$ is large enough, $N_{p}^{n}$ has a compact closure. So therefore have $V_{p}^{n} \cap N_{p}^{n}$ and (since $f$ is closed) $f\left(V_{p}^{n} \cap N_{p}^{n}\right)$. But, if $\operatorname{Fr}\left(F_{p}\right) \neq \varnothing, f\left(V_{p}^{n} \cap N_{p}^{n}\right)=f\left(V_{p}^{n}\right)=W_{n}(p)$. If $\operatorname{Fr}\left(F_{p}\right)=\varnothing, F_{p}$ is an open inverse set, so $(p)$ is a compact neighborhood of $p$. Finally, the proof for local separability is entirely similar.

Remark. Corollary 1 does not apply to local peripheral compactness. ${ }^{6}$

CoROllary 2. If $f$ is a closed continuous mapping of a metric space

6 A space is locally peripherally compact if every point has arbitrarily small neighborhoods with compact frontiers. Every 0-dimensional space is locally peripherally compact. 
$S$ onto a locally countably compact space $E$, then $E$ is metrizable (and consequently the sets $\operatorname{Fr}\left(f^{-1}(p)\right)$ are compact).

For each point $p$ of $E$ has a sequence of open neighborhoods $X_{n}(p)$ $=\left\{q \mid F_{q} \subset S\left(F_{p}, 1 / n\right)\right\}$ with the property $\bigcap_{n} X_{n}(p)=(p)$. Since $E$ is locally countably compact and regular, ${ }^{7}$ one easily derives open neighborhoods $Y_{n}(p)$ of $p$ such that $\mathrm{Cl}\left(Y_{n}(p)\right) \subset X_{n}(p), Y_{n}(p)$ $\supset Y_{n+1}(p)$, and $\mathrm{Cl}\left(Y_{1}(p)\right)$ is countably compact; here $\mathrm{Cl}$ denotes the closure. It is a straightforward matter to verify that the sets $Y_{n}(p)$ form a basis of neigborhoods at $p$; hence $E$ satisfies the first axiom of countability, and Theorem 1 applies.

3. Monotone mappings. The condition that $f$ be closed cannot be omitted in Theorem 1, in general [2, p. 70, Example 2], even if $f$ is open, $S$ a subset of the plane, $E$ a Hausdorff space with a countable basis, and the sets $f^{-1}(p)$ are all compact. However, in some cases the closedness of $f$ follows from the other hypotheses. This is so, for example, if $S$ is compact and $E$ is Hausdorff. A less obvious example is the following, which generalizes a theorem of A. V. Martin [5, Theorem $5]$.

THEOREM 2. Let $E$ be the hyperspace of a decomposition of a locally peripherally compact metric space $S$ into connected sets with compact frontiers; equivalently, let $E=f(S)$ where $f$ is a quasicompact continuous mapping such that, for each $p \in E, f^{-1}(p)$ is connected and $\operatorname{Fr}\left(f^{-1}(p)\right)$ is compact. Then, if $E$ is a Hausdorff space, $f$ is closed (and consequently $E$ is metrizable, by Theorem 1$)$; further, $E$ is then locally peripherally compact.

As before, we write $F_{p}=f^{-1}(p)$; we first prove that the decomposition $\left\{F_{p}\right\}$ of $S$ is upper semi-continuous. Given any open set $U \supset F_{p}$, cover $\operatorname{Fr}\left(F_{p}\right)$ by a finite number of open sets $U_{1}, U_{2}, \cdots, U_{m}$ such that $U_{i} \subset U$ and $\operatorname{Fr}\left(U_{i}\right)$ is compact $(1 \leqq i \leqq m)$. Let $V=U_{1} \cup U_{2}$ $\cup \ldots \cup U_{m} \cup \operatorname{Int}\left(F_{p}\right)$; then $F_{p} \subset V \subset U$, and the set Fr $(V)$, being a closed subset of $\operatorname{Fr}\left(U_{1}\right) \cup \ldots \cup \operatorname{Fr}\left(U_{m}\right) \cup \operatorname{Fr}\left(F_{p}\right)$, is compact. Take $W_{n}=S\left(\operatorname{Fr}\left(F_{p}\right), 1 / n\right) \cup \operatorname{Int}\left(F_{p}\right)$, an open set containing $F_{p}$; it is enough to prove that, for some $n$, every $F_{q}$ meeting $W_{n}$ is contained in $V$. Suppose this false; then, for each $n$, we obtain $q_{n} \in E$ such that $F_{q_{n}}$ meets both $W_{n}$ and $S-V$. Since $q_{n} \neq p$ (for $F_{p} \subset V$ ), $F_{q_{n}}$ contains a point $y_{n}$ of $S\left(\operatorname{Fr}\left(F_{p}\right), 1 / n\right)$, and there exists $x_{n} \in \operatorname{Fr}\left(F_{p}\right)$ such that $\rho\left(x_{n}, y_{n}\right)<1 / n$. Again, for all $n$ greater than some $n_{0}$, we have $1 / n<\rho\left(\operatorname{Fr}\left(F_{p}\right), S-V\right)$, so that $W_{n} \subset V$. Hence $F_{q_{n}}$ meets both $V$ and $S-V$, and so meets $\operatorname{Fr}(V)$, say in $z_{n}\left(n>n_{0}\right)$.

For a suitable subsequence of values $n^{\prime}$ of $n$, we have $z_{n^{\prime}} \rightarrow z \in \operatorname{Fr}(V)$,

\footnotetext{
${ }^{7}$ In fact, any closed continuous image of a normal space is normal [10].
} 
$x_{n^{\prime}} \rightarrow x \in \operatorname{Fr}\left(F_{p}\right)$, and therefore $y_{n^{\prime}} \rightarrow x$. Now $z \notin F_{p}$, since $V$ contains $F_{p}$ and is disjoint from $\operatorname{Fr}(V)$. Let $f(z)=q$; then $q \in E-(p)$ and $z \in F_{q}$. Since $E$ is a Hausdorff space, there exist disjoint open inverse sets $Y \supset F_{p}, Z \supset F_{q}$. If $n^{\prime}$ is large enough, $y_{n^{\prime}} \in Y$ and $z_{n^{\prime}} \in Z$; thus $F_{q_{n^{\prime}}}$ meets, and so is contained in, both $Y$ and $Z$, giving a contradiction.

This proves the decomposition upper semi-continuous; the mapping $f$ is therefore closed, and Theorem 1 applies. Finally, to prove $E$ locally peripherally compact, suppose $p \in E$ and an open set $G \ni p$ given; as above, we construct an open set $V \supset F_{p}$ such that $V \subset f^{-1}(G)$ and $\operatorname{Fr}(V)$ is compact. Let $W=\left\{q \mid F_{q} \subset V\right\}=E-f(S-V)$, an open set such that $p \in W \subset G$. Write $X=f^{-1}(W)$; clearly $X \subset V$. Suppose $q$ is any point of $\operatorname{Fr}(W)$; then $q \notin W$, so $F_{q}$ meets $S-V$. But $q \in \bar{W}$ $=\mathrm{Cl}(f(X))=f(\bar{X})$ (since $f$ is closed), so $q \in f(\bar{V})$ and $F_{q}$ also meets $\bar{V}$. Being connected, $F_{q}$ meets $\operatorname{Fr}(V)$; hence $q \in f(\operatorname{Fr}(V))$. This proves $\operatorname{Fr}(W) \subset f(\operatorname{Fr}(V))$, which is compact; thus $\operatorname{Fr}(W)$ is also compact, q.e.d.

4. General quasicompact mappings. The condition that the sets $F_{p}$ are all connected cannot be omitted from Theorem 2, even if they are all compact and $S$ is a locally compact subset of the plane; examples to show this are easily constructed. We have, however, the following theorem (which generalizes another theorem of Martin [5, Corollary to Theorem 4$]$ ), in which the mapping $f$ can in fact be neither open nor closed.

THEOREM 3. If $f$ is a quasicompact continuous mapping of a locally compact separable metric space $S$ onto a Hausdorff space $E$ with a locally countable basis, then $E$ is a locally compact separable metric space.

LeMma 1. Let $f$ be a quasicompact continuous mapping of a topological space $S$ onto a Hausdorff space $E$ with a locally countable basis at $p \in E$, and let $\left\{U_{n}\right\}(n=1,2, \cdots)$ be an increasing sequence of open sets of $S$ such that $\cup U_{n} \supset F_{p}=f^{-1}(p)$. Then, for some $n, p \in \operatorname{Int}\left(f\left(U_{n}\right)\right)$.

Let $\left\{W_{n}\right\}(n=1,2, \cdots)$ be a basis of neighborhoods of $p$; we may suppose $W_{1} \supset W_{2} \supset \ldots$. Without loss of generality, we may assume that each $U_{n}$ meets $F_{p}$. We show that, for some $n, f\left(U_{n}\right) \supset W_{n}$. Suppose not; then, for each $n$, there is a point $q_{n} \in W_{n}-f\left(U_{n}\right)$. Write $Q=\left\{q_{n}\right\} \quad(n=1,2, \cdots), X=f^{-1}(Q)$; as $p \in \bar{Q}-Q, Q$ is not closed, and therefore $X$ is not closed and there exists a point $x \in \bar{X}-X$. Then $f(x) \in \bar{Q}-Q$, and it is easy to deduce (from the fact that $E$ is Hausdorff and $\left.q_{n} \rightarrow p\right)$ that $f(x)=p$. Hence $x \in F_{p}$, so $x \in U_{n}$ for some $n$. Then $U_{n}-U\left\{f^{-1}\left(q_{m}\right), 1 \leqq m \leqq n\right\}$ is an open set containing $x$ but 
disjoint from $X$, contradicting $x \in \bar{X}$ and establishing the lemma.

LeMmA 2. If $E$ is the hyperspace of a decomposition $\left\{F_{p}\right\}$ of a locally compact topological space $S$, for which each $\mathrm{Fr}\left(F_{p}\right)$ is $\sigma$-compact, ${ }^{8}$ and if $E$ is Hausdorff and has a locally countable base, then $E$ is locally compact.

As usual, we use $f$ to denote the natural quasicompact mapping of $S$ onto $E$, so that $f^{-1}(p)=F_{p}$. By hypothesis, given $p \in E$, we can write $\operatorname{Fr}\left(F_{p}\right)=U K_{n}(n=1,2, \cdots)$ where $K_{n}$ is compact. Since $S$ is locally compact, $K_{n}$ can be covered by finitely many open sets with compact closures; in this way we obtain open sets $G_{n} \supset K_{n}$ such that $\mathrm{Cl}\left(G_{n}\right)$ is compact and $G_{1} \subset G_{2} \subset \ldots$ Applying Lemma 1 to $U_{n}=G_{n} \cup \operatorname{Int}\left(F_{p}\right)$, we have $p \in \operatorname{Int}\left(f\left(U_{n}\right)\right)$ for some $n$. If $\operatorname{Fr}\left(F_{p}\right) \neq \varnothing$, this gives $p \in \operatorname{Int}\left(f\left(G_{n}\right)\right) \subset f\left(\mathrm{Cl}\left(G_{n}\right)\right)$, which is compact and closed in the Hausdorff space $E$. If $\operatorname{Fr}\left(F_{p}\right)=\varnothing,(p)$ is a compact neighborhood of $p$.

LemMA 3. If $E$ is the hyperspace of a decomposition $\left\{F_{p}\right\}$ of a topological space $S$ with a countable base, and if $E$ is Hausdorff and has a locally countable base, then $E$ has a countable base.

(For upper semi-continuous decompositions, this was proved by Whyburn [10, Theorem 10].)

Let $B=\left\{B_{m}\right\}$ be a countable base of open sets of $S$; we prove $E$ has a base of the form $\{$ Int $(f(U))\}$ where $U$ is a finite union of sets of $B$. Given any $p \in E$ and any open set $G \ni p$, we have $F_{p} \subset f^{-1}(G)$, and can cover $F_{p}$ by a sequence of sets $B_{m_{1}}, B_{m_{2}}, \cdots$, of $B$, all satisfying $B_{m_{i}} \subset f^{-1}(G)$. Write $U_{n}=B_{m_{1}} \cup \ldots \cup B_{m_{n}}$; by Lemma 1 we have $p \in \operatorname{Int}\left(f\left(U_{n}\right)\right)$ for some $n$, where $f\left(U_{n}\right) \subset G$, and the lemma is proved.

Proof of Theorem 3 . As $S$ is here separable metric and locally compact, the same is true of each $\operatorname{Fr}\left(F_{p}\right)$, which is therefore $\sigma$ compact. By Lemma 2, $E$ is locally compact, and therefore regular. Also $E$ has a countable base, by Lemma 3 , and so it is metrizable.

As another immediate consequence of Lemma 3, we have:

COROLlARY. If $f$ is a quasicompact continuous mapping of a separable metric space $S$ onto a regular space $E$ with a locally countable base, then $E$ is separable metric.

The extension of Theorem 3 (and its corollary) to nonseparable spaces $S$ presents unexpected difficulties. We shall see from examples (\$6) that even an open continuous image of a locally compact metric space need not be metrizable, even if it is regular (and has a locally countable base) and the sets $\operatorname{Fr}\left(F_{p}\right)$ are compact. One fairly im-

${ }^{8} \mathrm{~A}$ set is $\sigma$-compact if it is the union of countably many compact sets. 
mediate extension of Theorem 3 is as follows.

THEOREM $3^{\prime}$. Let $f$ be a quasicompact continuous mapping of a locally compact metric space $S$ onto a Hausdorff space $E$ with a locally countable base, and suppose that the sets $\operatorname{Fr}\left(f^{-1}(p)\right)$ are separable and that $E$ is paracompact. Then $E$ is a locally compact metric space.

For the argument proving Theorem 3 here shows that each $p \in E$ has a compact neighborhood of the form $f(\bar{G})$ where $\bar{G}$ is compact. Since $E$ is Hausdorff, the mapping $f$ restricted to $\bar{G}$ is closed; hence, by a well-known special case of Theorem $1, f(\bar{G})$ is metrizable. Thus $E$ is locally metrizable. Since $E$ is paracompact, $E$ has a locally finite covering by closed metrizable sets, and is therefore [6] metrizable.

The condition in Theorem $3^{\prime}$ that $E$ be paracompact cannot be dispensed with, in general, even if $f$ is open (\$6). I do not know whether it is superfluous if the sets $f^{-1}(p)$ are assumed to be separable. If $f$ is open and the sets $f^{-1}(p)$ are separable, the paracompactness of $E$ follows from the other hypotheses (see the corollary to Theorem 4 below).

5. Open mappings. If $f$ is open, Theorem 3 has the following extension to not necessarily separable spaces; perhaps the most interesting feature of the theorem is that none of the hypotheses is superfluous (\$6).

THEOREM 4. If $f$ is an open continuous mapping of a metric, locally separable space $S$ onto a regular space $E$, and if for each $p \in E$ the set $f^{-1}(p)$ is separable, then $E$ is metrizable and locally separable.

Lемма 1. The theorem is true if $S$ is separable, and $E$ is then separable.

This is a special case of the corollary to Theorem 3 ; it is also obvious directly.

Lемма 2. If $f(S)=E$, where $f$ is open and continuous and each $f^{-1}(p)$ is separable, then for every separable subset $Y$ of $E, f^{-1}(Y)$ is separable.

Let $Q=\left\{q_{m}\right\} \quad(m=1,2, \cdots)$ be a countable subset of $Y$ such that $\bar{Q} \supset Y$, and for each $m$ let $X_{m}$ be a countable dense subset of $f^{-1}\left(q_{m}\right)$. Write $X=\bigcup X_{m}$; it is easy to see that the countable set $X$ is dense in $f^{-1}(Y)$.

Proof of Theorem 4. By a theorem of Alexandroff [1], $S$ can be expressed as a union of pairwise disjoint nonempty open sets $S_{\lambda}$, each of which is separable. Write $S_{\lambda} \sim S_{\mu}$ to mean that there exists a finite sequence $\lambda=\lambda_{0}, \lambda_{1}, \cdots, \lambda_{k}=\mu$ such that each set $f\left(S_{\lambda_{i-1}}\right)$ meets 
$f\left(S_{\lambda_{i}}\right)(i=1,2, \cdots, k)$. It is easily verified that $\sim$ is an equivalence relation. Let the union of the $S_{\mu}$ 's equivalent to $S_{\lambda}$ be $T_{\lambda}$; thus $T_{\lambda}$ is open, and $T_{\lambda}$ and $T_{\lambda^{\prime}}$ are either identical or disjoint. Further, $T_{\lambda}$ is an inverse set, (i.e., equals $f^{-1}\left(f\left(T_{\lambda}\right)\right.$ ). For suppose $F_{p}=f^{-1}(p)$ meets $T_{\lambda}$, say in $y \in F_{p} \cap S_{\mu}$ where $S_{\mu} \sim S_{\lambda}$. If $x$ is any point of $F_{p}$, we have $x \in$ some $S_{\nu}$ and $p \in f\left(S_{\nu}\right) \cap f\left(S_{\mu}\right)$; hence $S_{\nu} \sim S_{\mu} \sim S_{\lambda}$, so that $x \in T_{\lambda}$. Thus $F_{p} \subset T_{\lambda}$ whenever $F_{p}$ meets $T_{\lambda}$, and $T_{\lambda}$ is an inverse set. It follows that the distinct sets $f\left(T_{\lambda}\right)$ are disjoint and open, and they cover $E$; to prove the theorem, it will suffice to prove that each $f\left(T_{\lambda}\right)$ is separable metric, and by Lemma 1 it suffices to prove that each $T_{\lambda}$ is separable.

Now let $T_{\lambda}^{n}$ denote the union of those sets $S_{\mu}$ which can be reached from $S_{\lambda}$ in at most $n$ steps - that is, for which there is a sequence $\lambda=\lambda_{0}, \lambda_{1}, \cdots, \lambda_{k}=\mu$, of the type used to define $\sim$, with $k \leqq n$. Clearly $T_{\lambda}=T_{\lambda}^{0} \cup T_{\lambda}^{1} \cup \ldots$, and it is enough to prove that each $T_{\lambda}^{n}$ is separable. Suppose this true for one particular value of $n$. Then $T_{\lambda}^{n+1}$ consists of $T_{\lambda}^{n}$ together with those sets $S_{\mu}$ for which $f\left(S_{\mu}\right)$ meets $f\left(T_{\lambda}^{n}\right)$ - that is, for which $S_{\mu}$ meets $f^{-1}\left(f\left(T_{\lambda}^{n}\right)\right)$. By hypothesis, $T_{\lambda}^{n}$ is separable; hence so is $f\left(T_{\lambda}^{n}\right)$, and Lemma 2 now shows that $f^{-1}\left(f\left(T_{\lambda}^{n}\right)\right)$ is also separable. It can therefore meet at most countably many of the disjoint open sets $S_{\mu}$. Thus $T_{\lambda}^{n+1}$ is a union of countably many separable sets, and is again separable. Since $T_{\lambda}^{0}=S_{\lambda}$ and is separable, the separability of $T_{\lambda}^{n}$ follows for all $n$; and the theorem is proved.

CoROllary. If $f$ is an open continuous mapping of a locally compact metric space $S$ onto a Hausdorf space $E$, and if for each $p \in E$ the set $f^{-1}(p)$ is separable, then $E$ is a locally compact metric space. (Compare Theorem $3^{\prime}$.)

For, from the second lemma to Theorem $3, E$ is locally compact. and therefore regular; now Theorem 4 applies.

6. Counterexamples. The following examples show the need for the restrictions imposed in Theorems $3,3^{\prime}$ and $4 .^{9}$ In each case, $f$ will be an open (and so certainly quasi-compact) continuous mapping of a metric space $S$ onto a nonmetrizable space $E$; the openness of $f$ is easily verified from the fact that $E$ is in each case the hyperspace of a decomposition $\left\{F_{p}\right\}$ of $S$ which is lower semi-continuous (that is, given any open set $U$ meeting $F_{p}$, there exists an open set $V \supset F_{p}$ such that every $F_{q}$ meeting $V$ meets $U$ ). In the first two examples, $S$ is locally compact; in all three, the openness of $f$ guarantees that

\footnotetext{
'See $[2$, p. 70, Ex. 1, 2] for examples showing that "Hausdorff" cannot be replaced by " $T_{1}$ " in Theorems 3 and 3 ' (even if $S$ is compact), and that "regular" cannot be replaced by "Hausdorff" in Theorem 4 (even if $S$ is separable).
} 
$E$ will have a locally countable base.

(1) For each countable ordinal $\alpha$, let $E_{\alpha}$ denote the set of ordinals not exceeding $\alpha$; in the usual topology, $E_{\alpha}$ is a compact metric space, and we suppose it metrized with diameter $<1$, say with metric $\rho_{\alpha}$. Let $S$ be the set of all ordered pairs $(\alpha, \beta)$, where $\alpha, \beta$ are countable ordinals and $\alpha \geqq \beta$. Define a distance $\rho$ on $S$ by: $\rho\left\{(\alpha, \beta),\left(\alpha^{\prime}, \beta^{\prime}\right)\right\}$ $=\rho_{\alpha}\left(\beta, \beta^{\prime}\right)$ if $\alpha=\alpha^{\prime}, 1$ otherwise. Thus $S$ is the discrete union of the spaces $E_{\alpha}$, and is locally compact (all sets of diameter less than 1 having compact closures). The mapping $f$ given by $f(\alpha, \beta)=\beta$ is an open continuous mapping of $S$ onto the space $E$ of all countable ordinals (in its usual topology); the corresponding decomposition is $\left\{F_{\beta}\right\}$ where $F_{\beta}=\{(\alpha, \beta) \mid \alpha \geqq \beta\}$. Here $E$ is a completely normal Hausdorff space, and is locally compact and has a locally countable base, but is not metrizable.

(2) Our second example is similar, but $E$ will satisfy fewer separation axioms; to compensate for this, the sets $F_{p}$ will have compact frontiers. Let $I$ denote the unit interval $0 \leqq x \leqq 1$, and let $A, R$ denote respectively the sets of irrational and rational numbers in $I$. For each $a \in A$, choose one sequence $\left\{r_{n}(a)\right\} \rightarrow a$ with $r_{n}(a) \in R$ (e.g., by using the decimal expansion). Now take $S=(R \times A) \cup A$ metrized as follows: $\rho\left\{\left(r_{m}(a), a\right), a\right\}=1 / m, \rho\left\{\left(r_{m}(a), a\right),\left(r_{n}(a), a\right)\right\}=|1 / m-1 / n|$, and all other distances between distinct points are 1. Thus $S$ is the discrete union of sets $S_{a}=(R \times a) \cup(a)$, and $S_{a}$ consists of a sequence $\left\{\left(r_{m}(a), a\right)\right\}$ converging to $a$, together with a discrete sequence of points (the other points $(r, a))$. Clearly $S$ is a locally compact metric space (all closed sets of diameter less than 1 being compact). Now map $S$ in $I$ by: $f((r, a))=r, f(a)=a$. The corresponding decomposition is characterized by $F_{r}=\{(r, a) \mid a \in A\}, F_{a}=(a)$. Each $F_{r}(r \in R)$ is both open and closed in $S$, so in any case $\operatorname{Fr}\left(F_{p}\right)$ is compact $(p \in I)$ and has, in fact, at most one point. Further, from the fact that each $F_{p}$ is either open or consists of a single point, it is immediate that the decomposition $\left\{F_{p}\right\}$ is lower semi-continuous; thus $f$ is an open continuous mapping of $S$ onto the hyperspace $E$ of the decomposition, which is easily seen to consist of $I$ with the following topology. A typical neighborhood of $r \in R$ is $(r)$; a typical neighborhood of $a \in A$ consists of $a$ together with all points $r_{n}(a)$ for $n$ sufficiently large. These neighborhoods are closed, as well as open, in $E$; hence $E$ is a regular Hausdorff space. But $E$ is separable $(R$ is a countable dense set), yet contains an uncountable discrete set $(A)$; hence $E$ is not metrizable. (If the continuum hypothesis is true, $E$ cannot be normal; see F. B. Jones, Concerning normal and completely normal spaces, Bull. Amer. Math. Soc. vol. 43 (1937) pp. 671-677.)

(3) In our last example, the sets $F_{p}$ will themselves be compact, 
though $S$ will no longer be locally separable. Let $N=$ set of all positive integers, $G=$ set of all mappings $g$ of $N$ in $N$. Roughly, $S$ will consist of $N, G$, a sequence of points converging to each $g \in G$, and $c$ sequences of points (one for each $g \in G$ ) converging to each $n \in N$. Precisely, we take $S=G \cup(G \times N) \cup N \cup(N \times G \times N)$, and define a metric $\rho$ on $S$ as follows:

$$
\begin{gathered}
\rho\{g,(g, n)\}=\frac{1}{n} ; \quad \rho\{(g, n),(g, m)\}=\frac{1}{n}+\frac{1}{m} \quad \text { if } n \neq m ; \\
\rho\{n,(n, g, m)\}=\frac{1}{m} ; \rho\left\{(n, g, m),\left(n, g^{\prime}, m^{\prime}\right)\right\}=\frac{1}{m}+\frac{1}{m^{\prime}} \\
\text { if }(g, m) \neq\left(g^{\prime}, m^{\prime}\right) ;
\end{gathered}
$$

and all other distances between distinct points are 2 . It is easily verified that $\rho$ is a metric. Note that the points $(g, m)$ and $(n, g, m)$ are isolated, each having distance at least $1 / m$ from any other point. Decompose $S$ into the 2-point sets $F_{o n}=\{(g, n),(n, g, g(n))\}$ and the other single points. The decomposition is lower semi-continuous (for the sets $F_{p}$ with more than one point are open), and so corresponds to an open continuous mapping $f$ of $S$ onto the hyperspace $E$; further the sets $F_{p}\left(=f^{-1}(p)\right)$ are all compact (having, in fact, at most 2 points). It is a straightforward matter to verify that $E$ is a regular $T_{1}$ space. Now the sets $f(G), f(N)$ are disjoint and closed in $E$; if $E$ were normal, there would be disjoint open sets $U \supset f(N), V \supset f(G)$. Then $N \subset f^{-1}(U)$, which is open in $S$; hence, for each $n \in N$, we can choose $m=h(n)$ (say) large enough for $S(n, 1 /(m-1)) \subset f^{-1}(U)$, and this gives $f(n, g, h(n)) \in U$. This is true for all $g \in G$, and so in particular for $g=h$; thus $f(h, n)=f(n, h, h(n)) \in U$, for all $n \in N$. But if $n$ is large enough, $(h, n) \in S(h, 1 /(n-1)) \subset f^{-1}(V)$, so that $f(h, n) \in V$, contradicting $U \cap V=\varnothing$. Thus $E$ is not normal, and so certainly not metrizable. ${ }^{10}$

7. Open-closed mappings. We conclude by considering the case in which the mapping $f$ of the metric space $S$ is simultaneously open and closed-or, equivalently, in which the decomposition $\left\{F_{p}\right\}$ of $S$ is continuous (i.e., both upper and lower semi-continuous). The essential content of the following theorem is due to Wallace [8], but Theorem 1 makes the proof very simple.

\footnotetext{
${ }^{10}$ Incidentally, example (3) answers a question raised in [2, p. 70] by giving a decomposition of a normal space for which the hyperspace is regular but not normal. Another example answering this question has been given by T. Ganea, On the Prüfer manifold and a problem of Alexandroff and Hopf, Acta Scientiarum Math. vol. 15 (1954) pp. 231-235.
} 
THEOREM 5. Necessary and sufficient conditions for a decomposition of a metric space $S$ into disjoint nonempty closed sets $F_{p}$ to be continuous are:

(a) Each $F_{p}$ is either open or compact.

(b) Given a compact $F_{p}$ and $\epsilon>0$, there exists $\delta>0$ such that whenever $\rho\left(F_{p}, F_{q}\right)<\delta$ we have $d\left(F_{p}, F_{q}\right)<\epsilon$ (d denoting the Hausdorff distance).

To prove (a) and (b) necessary, we note that the decomposition space $E$ now has a locally countable basis (for the corresponding mapping $f$ of $S$ onto $E$ is open), so by Theorem 1 each $\operatorname{Fr}\left(F_{p}\right)$ is compact. If Int $\left(F_{p}\right)=\varnothing$, this proves $F_{p}$ compact. If Int $\left(F_{p}\right) \neq \varnothing$, $f\left(\operatorname{Int}\left(F_{p}\right)\right)=(p)$ is open in $E$, so $F_{p}$ is open in $S$. Thus (a) follows; and (b) is now a routine restatement of upper and lower semi-continuity. Conversely, if (a) and (b) are given, it is easy to deduce that the decomposition is both upper and lower semi-continuous.

Corollary." If $f(S)=E$, where $f$ is an open, closed, continuous mapping of a metric space $S$, then $E$ is metrizable, with metric $\sigma$ given by $\sigma(p, q)=d\left(f^{-1}(p), f^{-1}(q)\right)$.

\section{REFERENCES}

1. P. Alexandroff, Über die Metrization der im kleinen kompakten topologischen Räume, Math. Ann. vol. 92 (1924) pp. 294-301.

2. P. Alexandroff and H. Hopf, Topologie I, Berlin, 1935.

3. A. H. Frink, Distance functions and the metrization problem, Bull. Amer. Math. Soc. vol. 43 (1937) pp. 133-142.

4. S. Hanai, On closed mappings, Proc. Japan. Acad. vol. 30 (1954) pp. 285-288.

5. A. V. Martin, Decompositions and quasi-compact mappings, Duke Math. J. vol. 21 (1954) pp. 463-469.

6. J. Nagata, On a necessary and sufficient condition of metrizability, J. Inst. Polytech. Osaka City Univ. Ser. A vol. 1 (1950) pp. 93-100.

7. I. A. VaYnsteY̌n, On closed mappings of metric spaces, Doklady Akad. Nauk SSSR. N. S. vol. 57 (1947) pp. 319-321.

8. A. D. Wallace, Some characterizations of interior transformations, Amer. J. Math. vol. 61 (1939) pp. 757-763.

9. G. T. Whyburn, Analytic topology, New York, 1942.

10. - Open and closed mappings, Duke Math. J. vol. 17 (1950) pp. 69-74.

11. W. A. Wilson, On semi-metric spaces, Amer. J. Math. vol. 53 (1931) pp. 361373.

The UNIVERSITy, MANCHESTER

11 This corollary is due to V. K. Balanchandran, A mapping theorem for metric spaces, Duke Math. J. vol. 22 (1955) pp. 461-464. 